\title{
Supernovae, the Accelerating Cosmos, and Dark Energy
}

\section{Brian Schmidt}

Reserach School of Astronomy \& Astrophysics, Australia National University, Mount Stromlo Observatory, Canberra ACT 2611, Australia email: brian@mso.anu.edu.au

\begin{abstract}
Type Ia supernovae remain one of Astronomy's most precise tools for measuring distances in the Universe. I describe the cosmological application of these stellar explosions, and chronicle how they were used to discover an accelerating Universe in 1998 - an observation which is most simply explained if more than $70 \%$ of the Universe is made up of some previously undetected form of 'Dark Energy'. Over the intervening 13 years, a variety of experiments have been completed, and even more proposed to better constrain the source of the acceleration. I review the range of experiments, describing the current state of our understanding of the observed acceleration, and speculate about future progress in understanding Dark Energy.
\end{abstract}

Text of presentation not available. 